\title{
Inhibition of angiopoietin-1 (ANGPT1) affects vascular integrity in ovarian hyperstimulation syndrome (OHSS)
}

\author{
Leopoldina Scotti ${ }^{\mathrm{A}, \mathrm{D}}$, Dalhia Abramovich ${ }^{\mathrm{A}}$, Natalia Pascuali ${ }^{\mathrm{A}}$, \\ Luis Haro Durand $^{\mathrm{A}}$, Griselda Irusta $^{\mathrm{A}}$, Ignacio de Zúñiga ${ }^{\mathrm{B}}$, Marta Tesone $^{\mathrm{A}, \mathrm{C}}$ \\ and Fernanda Parborell ${ }^{\mathrm{A}}$ \\ A Instituto de Biología y Medicina Experimental (IByME) - CONICET, Vuelta de Obligado 2490 \\ (1428) Buenos Aires, Argentina. \\ ${ }^{B}$ Centro Médico PREGNA Medicina Reproductiva, Juncal 3490 (1425) Buenos Aires, Argentina. \\ ${ }^{C}$ Departamento de Química Biológica, Facultad de Ciencias Exactas y Naturales - Universidad de \\ Buenos Aires, Intendente Güiraldes 2160 (1428), Buenos Aires, Argentina. \\ DCorresponding author. Email: leopoldinascotti@gmail.com
}

\begin{abstract}
Ovarian hyperstimulation syndrome (OHSS) is a complication of ovarian stimulation with gonadotrophins following human chorionic gonadotrophin (hCG) administration. The relationship between hCG and OHSS is partly mediated via the production of angiogenic factors, such as vascular endothelial growth factor A (VEGFA) and angiopoietins (ANGPTs). Here, we investigated the effect of ANGPT1 inhibition on ovarian angiogenesis in follicular fluid (FF) from women at risk of OHSS, using the chorioallantoic membrane (CAM) of quail embryos as an experimental model. We also analysed cytoskeletal changes and endothelial junction protein expression induced by this FF in the presence or absence of an ANGPT1-neutralising antibody in endothelial cell cultures. The presence of this antibody restored the number of vascular branch points and integrin $\alpha_{\mathrm{v}} \beta_{3}$ levels in the CAMs to control values. ANGPT1 inhibition in FF from OHSS patients also restored the levels of claudin-5, vascular endothelial cadherin and phosphorylated $\beta$-catenin and partially reversed actin redistribution in endothelial cells. Our findings suggest that ANGPT1 increases pathophysiological angiogenesis in patients at risk of OHSS by acting on tight and adherens junction proteins. Elucidating the mechanisms by which ANGPT1 regulates vascular development and cell-cell junctions in OHSS will contribute to identifying new therapeutic targets for the treatment of human diseases with aberrant vascular leakage.
\end{abstract}

Additional keywords: angiogenesis, follicular fluid, ovary, reproduction.

Received 22 October 2013, accepted 11 September 2014, published online 11 November 2014

\section{Introduction}

Ovarian hyperstimulation syndrome (OHSS), an iatrogenic complication of ovulation induction and ovarian stimulation for assisted reproductive technology (ART), is characterised by cystic enlargement of the ovaries and rapid fluid shifts from the intravascular compartment to the third space. In its severe form, it is a potentially life-threatening condition that results in hospitalisation in $1.9 \%$ of cases (Humaidan et al. 2010). It has been recognised that human chorionic gonadotrophin (hCG) is the factor triggering the syndrome (Gómez et al. 2010). The pathophysiology of the syndrome is not completely understood and no specific therapy or prevention is available yet. The relationship between hCG and OHSS is thought to be mediated via the production of angiogenic factors. Many of these factors have been identified and comprise members of the vascular endothelial growth factor A (VEGFA) and basic fibroblast growth factor (FGF-2) families, transforming growth factors (TGFs), angiopoietins (ANGPTs) and platelet-derived growth factors
(PDGFs; Hanahan and Folkman 1996; Neufeld et al. 1999; Carmeliet 2000; Otrock et al. 2007). The angiogenic process, which culminates with the formation of structurally and functionally mature blood vessels, requires the synchronised action of numerous factors. Even though it is known that VEGFA is crucial for the initiation of angiogenesis, other factors, such as angiopoietins, are necessary for the stabilisation of newly developed capillaries (Suri et al. 1996; Maisonpierre et al. 1997). Both ANGPT1 and ANGPT2 bind to the tyrosine kinase receptor Tie-2 with the same affinity (Maisonpierre et al. 1997). While ANGPT1 induces Tie-2 phosphorylation, which triggers various biological effects, ANGPT2 acts as a natural antagonist of ANGPT1, since it binds to Tie-2 but does not phosphorylate the receptor (Maisonpierre et al. 1997). The ovarian expression of Vegfa, Angpt1 and Angpt2 mRNAs has been reported in many species, such as rats (Phillips et al. 1990; Koos 1995; Maisonpierre et al. 1997), cows (Goede et al. 1998) and nonhuman primates (Hazzard et al. 1999). These studies propose 
that these angiogenic factors have a key role in ovarian angiogenesis.

We have previously studied the effect of VEGF or ANGPT1 inhibition in the rat ovary by intrabursal administration of the VEGFA inhibitor, TRAP, or a neutralising antibody against ANGPT1, respectively. We demonstrated in these models that there is an increase in the number of atretic follicles, caused by changes in the ratio of pro-apoptotic to anti-apoptotic proteins (Abramovich et al. 2006; Parborell et al. 2008).

Furthermore, we have shown that while ANGPT1 concentration in follicular fluid (FF) from women at risk of developing OHSS is higher than in FF from control patients, the levels of the soluble form of the receptor Tie-2 (sTie-2) remain unchanged. In addition, the incubation of endothelial cells with FF from patients at high risk of OHSS increases their migration significantly compared with that in the presence of FF from control patients. The inhibition of ANGPT1 in FF from OHSS patients by the use of a neutralising antibody decreases the migration of endothelial cells exposed to FF from OHSS women (Scotti et al. 2013).

As mentioned above, OHSS is characterised by increased capillary permeability that leads to leakage of fluid from the vascular compartment (Rodewald et al. 2009). The regulation of vascular permeability involves endothelial cell-to-cell junctions, such as tight (TJ) and adherens junctions (AJ). Tight junctions, which are composed of at least three different families of transmembrane proteins (claudins, occludins and junction adhesion molecules), form a barrier to molecule diffusion from the vessel lumen to the tissue parenchyma (Groten et al. 2006). Claudin-5, which is specific to endothelium (Rodewald et al. 2009), is one of the main claudins. Claudin-5-deficient mice die shortly after birth due to a defective blood-brain barrier function (Herr et al. 2013). Adherens junctions involve transmembrane proteins belonging to the cadherin family. Vascular endothelial cadherin (VE-cadherin) is one of the main adhesion molecules of AJ. VE-cadherin links to the actin filament network through proteins called catenins (Villasante et al. 2007). In mice, VEcadherin deficiency causes embryonic lethality, with endothelial cells unable to form vascular structures (Carmeliet et al. 1999).

Several authors have shown that the ovary requires tightly controlled dynamic changes in the localisation and expression of adhesion molecules (Groten et al. 2006; Rodewald et al. 2007). As described by Rodewald et al. (2007), the expression of TJ proteins is differentially regulated during follicular development, which suggests that these proteins play an important part in folliculogenesis and ovarian angiogenesis. Furthermore, it is known that in female angiogenesis-related reproductive disorders such as OHSS, VEGFA is responsible for an increase in endothelial migration and vascular permeability. As reported by Villasante et al. (2007), the effect of VEGFA is mediated, at least partly, through the modulation of VE-cadherin function. The changes induced in VE-cadherin and the subsequent loss of endothelial barrier architecture are impaired by VEGFA inhibition (Villasante et al. 2007).

So far, no study has focussed on the effect of ANGPT1, another major angiogenic factor, on $\mathrm{TJ}$ and $\mathrm{AJ}$ in OHSS. Therefore, the aim of the present study was to investigate the effect of FF from women at risk of OHSS, in the presence of an
ANGPT1-neutralising antibody, on the chorioallantoic membrane (CAM) of quail embryos as an experimental model of in vivo angiogenesis. We also analysed the effect of $\mathrm{FF}$ in the presence of the ANGPT1-neutralising antibody on cytoskeletal changes and on TJ and AJ protein expression of endothelial cells, so as to provide more information regarding the increased endothelial permeability in OHSS.

\section{Materials and methods}

\section{Subjects and collection of FF}

This study was approved by the ethics committee of the Institute of Biology and Experimental Medicine (IByME, Buenos Aires, Argentina). FF was obtained from 39 patients (25-41 years old) undergoing assisted reproductive technology procedures at the Reproductive Medicine Center PREGNA (Buenos Aires, Argentina). All the patients provided written informed consent before recruitment.

In this study, patients with pelvic pathologies, such as pelvic inflammatory disease, endometriosis or uterine fibroids, were excluded. Patients were classified into two groups: the control group $(n=20)$ and the OHSS group $(n=19)$, and matched for age.

To consider a patient at risk of OHSS, these criteria were followed: serum oestradiol level $>3000 \mathrm{pg} \mathrm{mL}^{-1}$ on the day of hCG administration and retrieval of $>20$ oocytes (Navot et al. 1992; Enskog et al. 1999; Orvieto 2013). When follicles reached $17 \mathrm{~mm}$, hCG (5000 IU mL ${ }^{-1}$ Pregnyl; Organon SA, Peteaux, France) was injected to induce ovulation. Oocyte retrieval was conducted $34 \mathrm{~h}$ later under vaginal ultrasound guidance, during which FF from 16-20-mm follicles was pooled. Only macroscopically clear samples of FF, without visible blood, were included in the study. After aspiration the FF was centrifuged for $10 \mathrm{~min}$ at $2000 \mathrm{~g}$ at $4^{\circ} \mathrm{C}$ to remove the debris and cellular components. The supernatant was then transferred to sterile tubes and stored at $-80^{\circ} \mathrm{C}$ until assayed.

For immunoneutralisation, the monoclonal anti-human ANGPT1 antibody (ab49694; Abcam, Cambridge, UK) was added to $\mathrm{FF}$ at an antibody: angiogenic factor molar ratio of $1000: 1$ and incubated for $1 \mathrm{~h}$ at $37^{\circ} \mathrm{C}$ (von Otte et al. 2006). This antibody shows no cross-reactivity with recombinant human angiopoietin-2. The anti-human IgG antibody (ab77118; Abcam) was used as control. To confirm the efficiency of the neutralising antibody, a western blot was performed to detect the phosphorylated form of ANGPT1 tyrosine kinase receptor, Tie-2 (ab78142; Abcam). This antibody recognises Tie-2 only when it is phosphorylated on Tyr992 (phospho Y992). Phospho Y992 regulates endothelial cell proliferation, migration and differentiation in endothelial cells during blood vessel formation (Davis et al. 1996; Fiedler and Augustin 2006; Bai et al. 2009). Pretreatment with the ANGPT1 antibody in both FF from control patients and FF from patients with high risk of OHSS resulted in $85 \%$ inhibition of Tie-2 phosphorylation (data not shown).

\section{In vivo angiogenesis: quail chorioallantoic membrane} (CAM) assay

Fertilised eggs of Japanese quail (Coturnix coturnix japonica; $n=180$ ) were purchased from a local farmer and incubated 
in ovo at $37^{\circ} \mathrm{C}$ and $60 \%$ relative humidity for 2 days. The CAM assay was performed according to the methodology described by Parsons-Wingerter et al. (1998) with modifications. Briefly, each egg was cleaned with $70 \%$ ethanol, then a hole was drilled through its shell at the acute pole of the egg and $1 \mathrm{~mL}$ albumen was removed with a sterile syringe. Afterwards, eggs were fenestrated with the aid of small dissecting scissors and embryos were cultured ex ovo in a 6-well plate for 5 days. Viability controls were performed every $48 \mathrm{~h}$.

Filter disks impregnated with FF from either control patients $(n=20)$ or patients at risk of OHSS $(n=19)$, with or without the antibody against ANGPT1, were placed on CAMs from 7-day embryos. The embryos were further incubated at $37^{\circ} \mathrm{C}$ for 2 days and then sacrificed by freezing. CAMs from 9-day-old embryos were fixed using a solution of $4 \%$ paraformaldehyde, $2 \%$ glutaraldehyde in phosphate-buffered saline (PBS) and processed as indicated by Brooks et al. (1998). Eggs treated with Hank's balanced salt solution only were used as the negative control.

The CAMs were dissected from the embryos and placed on slides. Angiogenesis was quantified by counting the number of blood vessel branch points on a delimited surface of CAMs under a stereomicroscope $(30 \times$; Leica Camera, Solms, Germany). The number of blood vessel branch points was counted within the area encompassing the entire disk.

\section{Enzyme-linked immunosorbent assay (ELISA)}

An ELISA was performed to measure the levels of integrin $\alpha_{\mathrm{v}} \beta_{3}$, a specific marker of angiogenic vascular tissues, in CAMs after treatment with FF from patients of both groups (Brooks et al. 1994; Eliceiri et al. 1998; Avraamides et al. 2008). Briefly, sections of CAMs confined to the disks were removed and homogenised in PBS. Each well of a 96-well plastic dish was coated in triplicate with $10 \mu \mathrm{g}$ of total protein, as determined by the Bradford assay. The plates were dried at $37^{\circ} \mathrm{C}$, blocked in PBS with $5 \%$ bovine serum albumin (BSA), incubated with anti$a_{\mathrm{v}} \beta_{3}$ antibody (LM609, 1:1000; Millipore Corp., Billerica, MA, USA), washed with PBS, incubated with anti-mouse IgG-peroxidase antibody produced in rabbit (Sigma-Aldrich Co., St Louis, MO, USA) and developed in 3,3', 5, $5^{\prime}$-tetramethylbenzidine (Sigma-Aldrich). Plates were read in an ELISA reader at $492 \mathrm{~nm}$.

\section{Endothelial cell culture}

EA.hy926 cells (a human umbilical vein cell line) were donated by Dr Gareth Owen (Pontifical Catholic University of Chile). This cell line was obtained by hybridisation of human umbilical vein endothelial cells (HUVEC) with the A549/8 human lung carcinoma cell line. In vitro, it has preserved highly differentiated functions that are characteristic of the human endothelial cell phenotype. Moreover, as with any cell line, it provides the advantages of stability through passage number, immortality and reproducibility of results (Edgell et al. 1983, 1990). Cells were maintained at $37^{\circ} \mathrm{C}$ with $5 \% \mathrm{CO}_{2}$ in Iscove's modified Dulbecco's medium (IMDM; Invitrogen Corp., Carlsbad, CA, USA) with $10 \%$ fetal bovine serum (FBS), $100 \mathrm{mg} \mathrm{mL}^{-1}$ streptomycin sulfate and $100 \mathrm{U} \mathrm{mL}^{-1}$ penicillin G. EA.hy926 cells were detached by $0.25 \%$ trypsin-ethylenediamine tetraacetic acid (EDTA), resuspended in the same medium and used for subsequent assays.

\section{Confocal microscopy analysis of actin filaments}

EA.hy926 cells were cultured to confluence on glass coverslips and incubated with FF (25\%) from patients for $24 \mathrm{~h}$ in the presence or absence of the ANGPT1 antibody (Scotti et al. 2013). The cells were washed with PBS, fixed for $5 \mathrm{~min}$ in $3.7 \%$ formaldehyde solution in PBS and permeabilised with $0.1 \%$ TRITON X-100 in PBS for 5 min. The cells were then stained with $50 \mu \mathrm{g} \mathrm{mL}^{-1}$ of TRITC-phalloidin (Sigma-Aldrich) for $40 \mathrm{~min}$ at room temperature. The excess of phalloidin was eliminated by washing with PBS. The labelled preparations were mounted on glass-slides using Vectashield mounting medium (Vector Laboratories, Inc., Burlingame, CA, USA) and observed in a confocal laser-scanning system attached to a microscope. All experiments were performed in triplicate and repeated three times.

\section{Western blot}

EA.hy926 cells were plated at a density of $5 \times 10^{5}$ cells per well in a 24-well plate, grown to confluence and incubated with FF $(25 \%)$ from both control patients and patients at risk of OHSS for $24 \mathrm{~h}$ or $10 \mathrm{~min}$ at $37^{\circ} \mathrm{C}$ with or without the antibody against ANGPT1 (Control patients $n=20$, OHSS patients $n=19$ ). Cells were washed with ice-cold PBS and lysed in homogenisation buffer containing $20 \mathrm{mM}$ TRIS-HCl pH $8,10 \%$ glycerol, $137 \mathrm{mM} \mathrm{NaCl}$ and supplemented with phosphatase inhibitors $(0.2 \mathrm{mM}$ sodium orthovanadate, $25 \mathrm{mM}$ sodium fluoride and $10 \mathrm{mM} \beta$-glycerophosphate) as well as protease inhibitors $(0.025 \mathrm{mM} \mathrm{N}$ - $p$-tosyl-lysine chloromethyl ketone, $0.5 \mathrm{mM}$ phenylmethanesulfonylfluoride (PMSF); $0.025 \mathrm{mM} \mathrm{N-CBZ-}$ L-phenylalanine chloromethyl ketone and $0.025 \mathrm{mM} \mathrm{L-1-}$ tosylamide-2-phenyl-ethylchloromethyl ketone). Samples were centrifuged at $10000 \mathrm{~g}$ for $10 \mathrm{~min}$ at $4^{\circ} \mathrm{C}$, after which the supernatants were collected and protein concentration was measured by the Bradford assay. After boiling for $5 \mathrm{~min}, 20 \mu \mathrm{g}$ of protein from each sample was loaded onto a polyacrylamide gel with sodium dodecyl sulfate (SDS; $10-15 \%)$ and electrophoresis was performed for $90 \mathrm{~min}$ at $25 \mathrm{~mA}$. The resolved proteins were transferred for $2 \mathrm{~h}$ onto nitrocellulose membranes and then blocked for $1 \mathrm{~h}$ at room temperature (blocking buffer: $5 \%$ nonfat milk, $0.05 \%$ Tween- 20 in $20 \mathrm{mM}$ Tris-buffered saline (TBS) $\mathrm{pH} 8.0$ ). The blots were incubated with appropriate primary antibodies: claudin-5 (35-2500, 1:2000; Invitrogen Corp.), zona occludens protein 1 (ZO-1; AB2272, 1:2000; Millipore), VE-cadherin (MAB1002, 1 : 1000; RandD Systems, Inc., Minneapolis, MN, USA) and phospho-b-catenin (4176, 1 : 1000; Cell Signalling Technology, Inc., Danvers, MA, USA) at $4{ }^{\circ} \mathrm{C}$. Loading was evaluated by Ponceau-S staining and normalisation to $\beta$-actin. Protein levels were analysed by densitometry with the Scion Image for Windows software (Scion Corporation, Frederick, MD, USA). Optical density data are expressed as arbitrary units \pm s.e.m.

\section{Statistical analysis}

All experiments were performed in triplicate and data are expressed as the mean \pm s.e.m. of at least three independent 
experiments using 20 control patients and 19 patients at risk of OHSS. Differences between groups were tested for significance using one-way ANOVA, followed by Tukey's test or the multiple-comparison Newman-Keuls test. Statistical significance was defined as $P<0.05$. For statistical analysis of data, we used the statistical program Prism Ver. 5.0 (GraphPad Software, Inc., La Jolla, CA, USA).

\section{Results}

\section{Patients' characteristics}

The number of oocytes retrieved and the serum levels of oestradiol at the time of hCG administration, but not the pregnancy rates, were different between the OHSS and control groups (Table 1).

\section{Effect of ANGPT1 in FF from patients at risk of OHSS on ovarian angiogenesis}

We evaluated the effect of FF from patients at risk of OHSS in the presence of an ANGPT1-neutralising antibody using the CAM assay, in an in vivo angiogenesis model. The CAM vasculature was quantified by the use of the morphometric pointcount method. FF from patients at risk of OHSS significantly increased the number of vascular branch points in the CAMs compared with control patients $(P<0.05)$. The presence of a neutralising antibody against ANGPT1 in the FF from OHSS patients restored such levels to control values (Fig. 1).

For biochemical studies, such as the determination of integrin $\alpha_{v} \beta_{3}$ by ELISA, unfixed CAMs were used. The results showed a significant increase in integrin $\alpha_{v} \beta_{3}$ levels in CAMs incubated with FF from OHSS patients in comparison with control patients $(P<0.05)$. Blocking ANGPT1 in FF from OHSS patients decreased the levels of integrin $\alpha_{\mathrm{v}} \beta_{3}$ in CAMs $(P<0.05)$ (Fig. 2).

\section{Effect of ANGPT1 in FF from patients at risk of OHSS on actin polymerisation of endothelial cells}

Using fluorescent microscopy we evaluated actin filament organisation after adding TRITC-phalloidin to cells cultured under different conditions (Fig. 3). The results showed that the incubation of endothelial cells with FF from patients at risk of OHSS caused a change in the cellular shape due to the rearrangement of actin filaments, evidenced by an irregular alignment of the filaments within the cells, compared with the FF from control patients. In addition, paracellular gap formation was induced in endothelial cells incubated with FF from OHSS

Table 1. Characteristics of control and ovarian hyperstimulation syndrome (OHSS) patients

Values are mean \pm s.e.m. NS, not significant

\begin{tabular}{lccc}
\hline Parameter & Control $(n=20)$ & OHSS $(n=19)$ & $P$ value \\
\hline Age (years) & $34.22 \pm 0.78$ & $32.62 \pm 0.67$ & NS \\
Retrieved oocytes $(n)$ & $10.75 \pm 0.84$ & $28.63 \pm 1.99$ & $<0.0001$ \\
Serum oestradiol $\left(\mathrm{pg} \mathrm{mL}^{-1}\right)$ & $1919 \pm 203$ & $3453 \pm 133$ & $<0.0001$ \\
Clinical pregnancy rate $(\%)$ & 52 & 57 & $\mathrm{NS}$ \\
\hline
\end{tabular}

patients compared with FF from control patients. In the OHSS group, stress fibres reorganised into a thickened cortical band near the plasma membrane with spike-like mesh structures extending into the cytosol (Fig. 3c). Moreover, actin fibres formed the perinuclear actin cap, which was connected to the nucleus envelope. The pre-incubation of FF from patients at risk of OHSS with the ANGPT1-neutralising antibody partially reversed the actin redistribution compared with the OHSS group without treatment (Fig. 3d).

\section{Effect of ANGPT1 in follicular fluid from patients at risk of OHSS on the expression of tight junction proteins}

We evaluated the expression of the tight junction proteins claudin-5 and zona occludens protein $1(\mathrm{ZO}-1)$ in endothelial

(a)

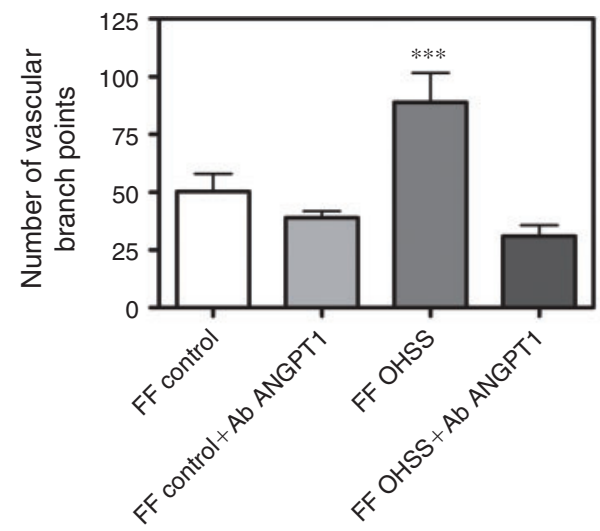

(b)
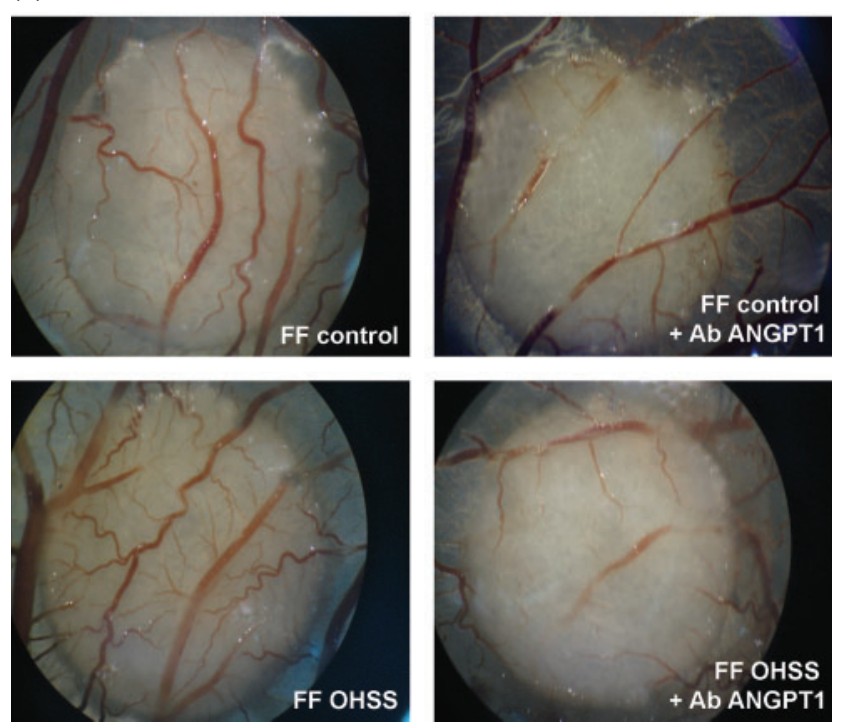

Fig. 1. Effect of follicular fluid (FF) from patients at risk of OHSS in the presence of an ANGPT1-neutralising antibody on ovarian angiogenesis. (a) Quantification of blood vessel branch points in a CAM assay. Data are expressed as means \pm s.e.m. of three independent experiments, using 20 control patients and 19 OHSS patients at risk of OHSS $(* * * P<0.001)$. (b) Representative images under a stereomicroscope of CAMs incubated with FF from control patients, FF from patients at risk of OHSS and FF from patients at risk of OHSS with the ANGPT1 antibody. 


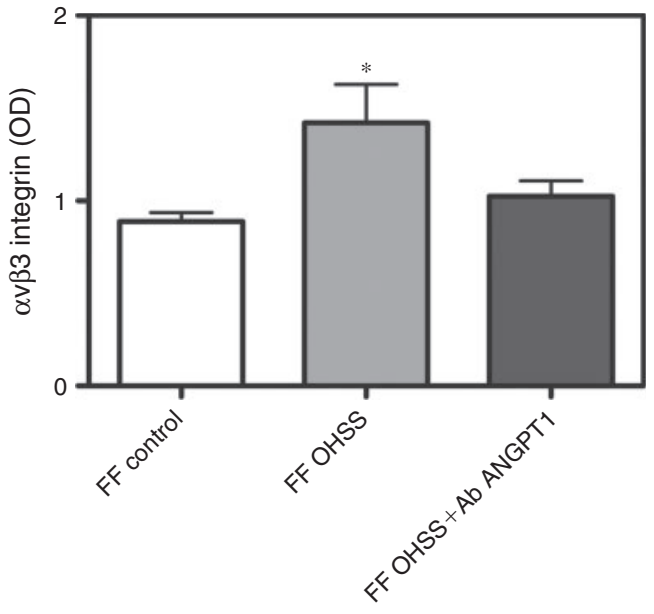

Fig. 2. Effect of follicular fluid (FF) from patients at risk of OHSS in the presence of an ANGPT1-neutralising antibody on the levels of integrin $\alpha_{v} \beta_{3}$ in CAMs. Data are expressed as means \pm s.e.m. of three independent experiments using 20 control patients and 19 OHSS patients at risk of OHSS $(* P<0.05)$. cells incubated with FF from OHSS patients, either alone or preincubated with the antibody against ANGPT1.

Claudin-5 levels in endothelial cells incubated with FF from OHSS patients were lower than those of the control group $(P<0.05)$ but higher in the presence of the antibody against ANGPT1 than in its absence $(P<0.01$; Fig. $4 a)$.

$\mathrm{ZO}-1$ levels in endothelial cells incubated with FF from OHSS patients were lower than those of the control group $(P<0.01$; Fig. 4b). No significant differences were observed with pre-incubation with and without the antibody against ANGPT1 (Fig. 4b).

Effect of ANGPT1 in FF from patients at risk of OHSS on the expression of adherens junction proteins

We next analysed the expression of VE-cadherin and phospho$\beta$-catenin (active form) proteins in endothelial cells incubated with FF from patients at risk of OHSS either alone or preincubated with the antibody against ANGPT1. VE-cadherin levels in endothelial cells incubated with FF from OHSS patients decreased as compared with the control group $(P<0.05)$ but were restored in the presence of the antibody
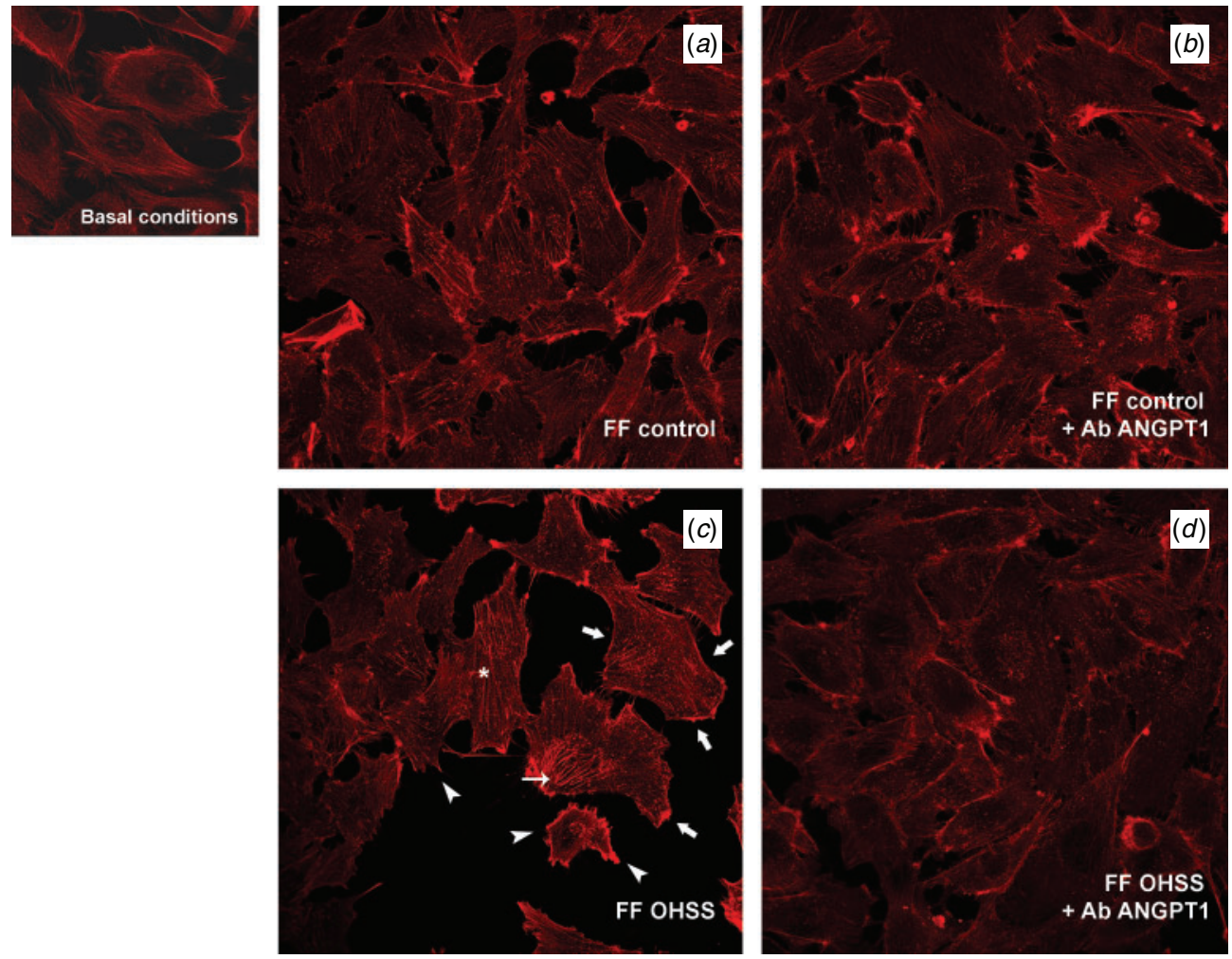

Fig. 3. Morphological changes in the organisation of endothelial cell actin cytoskeleton in the presence of follicular fluid (FF) from patients at risk of OHSS with an ANGPT1-neutralising antibody. Representative images of actin filaments stained with TRITC-phalloidin in endothelial cells incubated with (a) FF from control patients, $(b) \mathrm{FF}$ from control patients with the ANGPT1 antibody, (c) FF from patients at risk of OHSS and (d) FF from patients at risk of OHSS with the ANGPT1 antibody. Arrow heads indicate paracellular gaps, wide arrows indicate cortical band, narrow arrows indicate perinuclear actin cap and asterisks indicate stress fibres. Basal conditions are EA.hy926 cells in culture medium alone. Original magnification $60 \times$. 

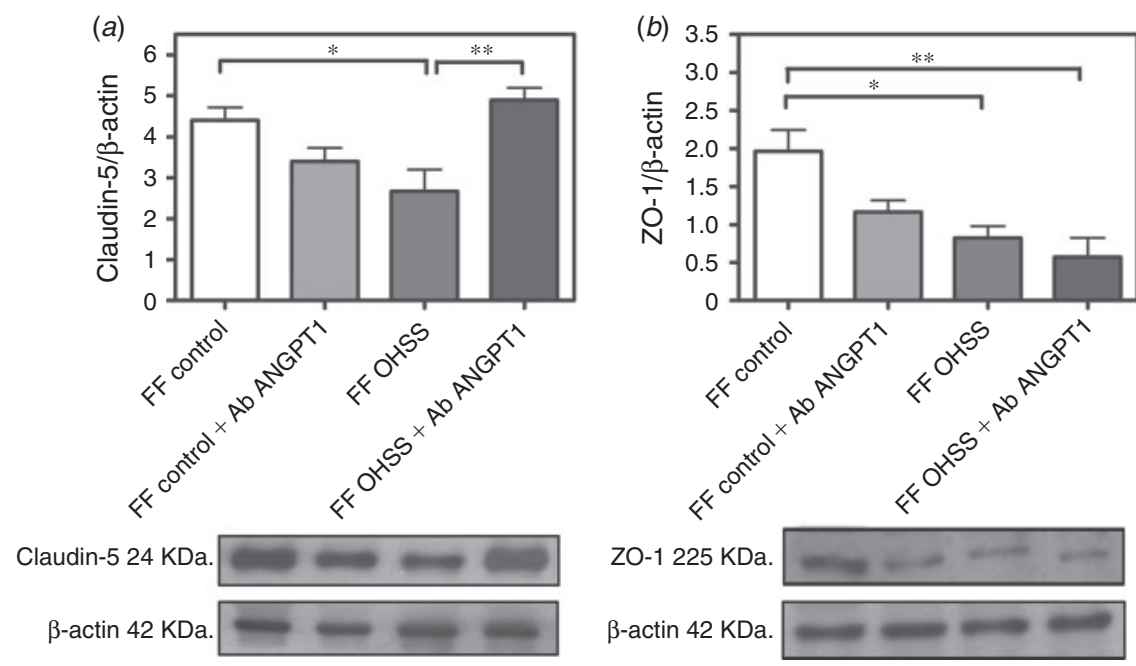

Fig. 4. Effect of follicular fluid (FF) from patients at risk of OHSS in the presence of an ANGPT1neutralising antibody on the expression of tight junction proteins. (a) Densitometric quantification of claudin-5. (b) Densitometric quantification of ZO-1. Representative immunoblots are shown in the lower panel. Data are expressed as means \pm s.e.m. normalised to $\beta$-actin of three independent experiments using 20 control patients and 19 OHSS patients at risk of OHSS $\left(* P<0.05,{ }^{*} P<0.01\right)$.
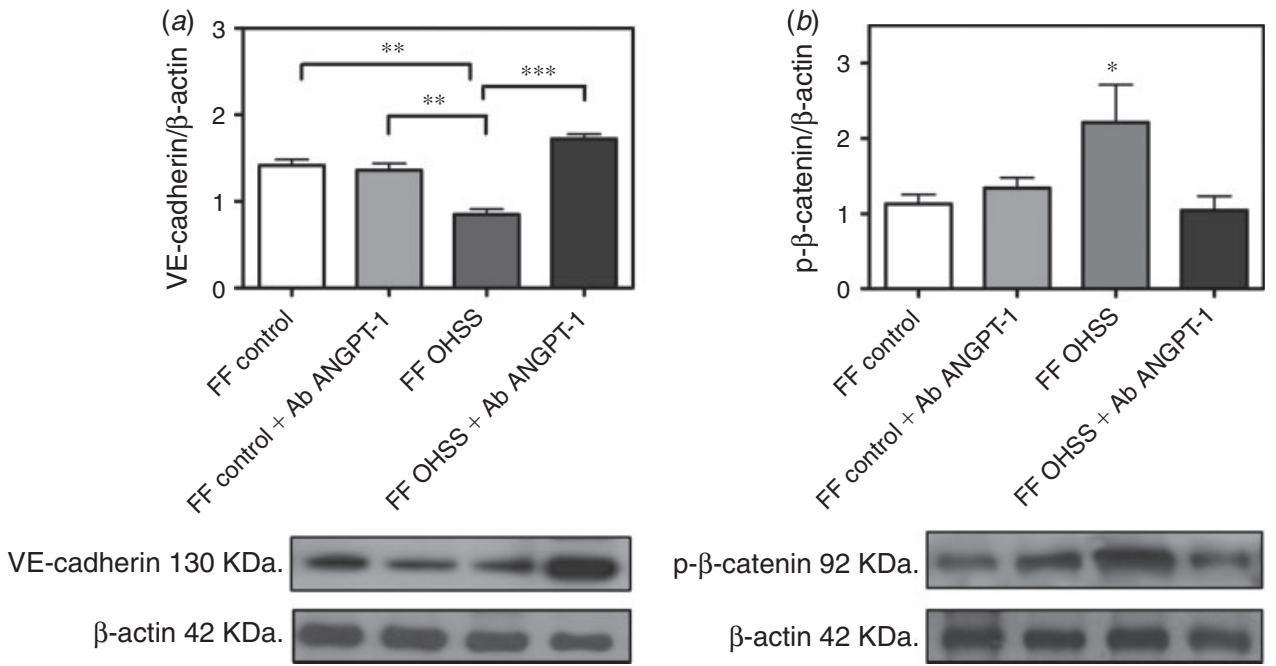

Fig. 5. Effect of follicular fluid (FF) from patients at risk of OHSS in the presence of an ANGPT1-neutralising antibody on the expression of adherens junction proteins. (a) Densitometric quantification of VE-cadherin. (b) Densitometric quantification of phospho- $\beta$-catenin. Representative immunoblots are shown in the lower panel. Data are expressed as means \pm s.e.m. normalised to $\beta$-actin of three independent experiments using 20 control patients and 19 OHSS patients at risk of OHSS $\left({ }^{*} P<0.05,{ }^{* *} P<0.01,{ }^{* * *} P<0.001\right)$.

against ANGPT1 $(P<0.001$; Fig. 5a). Phospho- $\beta$-catenin levels in endothelial cells incubated with FF from OHSS patients increased compared with the control group $(P<0.05)$ and were restored to normal values in the presence of the antibody against ANGPT1 $(P<0.05$; Fig. $5 b)$.

\section{Discussion}

The main features of OHSS include marked ovarian enlargement due to ovarian stimulation and overproduction of ovarian hormones and angiogenic substances, which contribute to an increase in capillary membrane permeability. This is the first study that, by means of in vivo and in vitro techniques, shows that ANGPT1 affects the development of vascular branches, distribution of actin filaments and expression of endothelial cell-cell junction proteins in an ovarian disorder such as OHSS.

The CAM assay is an intermediate stage between in vitro culture and in vivo animal experiments (Isachenko et al. 2012) and is widely used to study angiogenesis in tumour (Bérubé et al. 2005) and endometrial tissues (Nap et al. 2004). CAMs have 
also been used for the study of human skin culture (Kunzi-Rapp et al. 1999), for retinal surgery research (Leng et al. 2004) and for testing different biomaterials in tissue engineering (Valdes et al. 2002). It is worth mentioning that this assay could be applied to the analysis of angiogenic alterations in other female reproductive disorders, such as polycystic ovarian syndrome, by using FF from patients. In this study, we used the CAM system in Japanese quail to evaluate angiogenesis in OHSS. We observed that FF from patients at risk of OHSS increased the angiogenic activity in the CAMs compared with FF from control patients, as evidenced by the experiment performed in the presence of the ANGPT1-neutralising antibody. Additionally, we observed increased levels of integrin $\alpha_{\mathrm{v}} \beta_{3}$ in CAMs incubated with FF from OHSS patients in comparison with control patients. However, the inhibition of ANGPT1 in FF from OHSS patients decreased the levels of integrin $\alpha_{v} \beta_{3}$ in CAMs. These results suggest that the increased levels of ANGPT1 in FF from OHSS patients observed in our previous study (Scotti et al. 2013) could be partly responsible for the dysregulation of the vascular system balance.

Integrity of the cytoskeleton is critical for the endothelial barrier. Increased endothelial permeability to solutes and water is dependent on the shape of endothelial cells, which in turn is determined by reorganisation of actin filaments (Malik et al. 1989) and alterations in endothelial junction proteins (Maruo et al. 1992). An important regulatory mechanism for cell junction integrity is the distribution of actin to a peripheral pattern, leading to stress fibre formation. In this study, we observed that FF from patients at risk of OHSS induced strong actin filament remodelling, forming stress fibres. Pre-incubation of FF with the ANGPT1-neutralising antibody decreased this actin redistribution in endothelial cells. These results suggest that the ANGPT1 content in FF from these patients is partially responsible for the morphological change within endothelial cells. These results are consistent with data obtained previously by other authors and by our laboratory (Chen et al. 2010; Scotti et al. 2013). Chen et al. (2010) showed that FF from OHSS women remarkably induces actin redistribution, in comparison with the morphology of control endothelial cells in culture medium. Besides, we have previously observed that the incubation of FF from women at risk of OHSS with an ANGPT1neutralising antibody decreases endothelial cell migration (Scotti et al. 2013), considering that actin reorganisation is a prerequisite for cell migration and angiogenesis (Lauffenburger and Horwitz 1996; Menager et al. 1999).

As mentioned above, an endothelial hyperpermeability is generally accompanied by reorganisation of junction proteins, prompting a transient opening of the endothelial junctions and a subsequent increase in paracellular permeability. Thus, on the basis of this concept, we propose that TJ and AJ proteins are downstream targets of ANGPT1 in endothelial cells. Endothelial TJ constitute $\sim 20 \%$ of the total endothelial junctional complexes (Wójciak-Stothard et al. 2001). Claudin-5 is one of the main TJ transmembrane proteins and can form homotypic or heterotypic binding (Lampugnani 2012). The peripheral membrane adaptor protein ZO-1 links the carboxyl terminal of transmembrane adhesion proteins with $\alpha$-catenin, spectrin and F-actin. ZO-1 and ZO-2, but not ZO-3, are crucial for clustering of claudins, strand formation and barrier function (Umeda et al. 2006). ZO-1 provides an initial scaffold for TJ complexes, since it is recruited to early cadherin-containing contacts between endothelial cells. Therefore, formation of AJ favours the assembly of TJ (Itoh et al. 1997; Hartsock and Nelson 2008). In addition, several studies have shown that claudin-5 and ZO-1 play a crucial role in TJ regulation (Kanda et al. 2004; Jiao et al. 2011; Zehendner et al. 2011).

In this study, we observed that claudin-5 levels decreased significantly in endothelial cells incubated with FF from OHSS patients compared with control patients. The inhibition of ANGPT1 caused an increase in claudin-5 levels compared with FF from patients at risk of OHSS without treatment. These results suggest that, in patients at risk of OHSS, ANGPT1 could be partly responsible for the decrease in claudin-5 expression. Also, our results agree with published observations by Kitajima et al. (2006), who suggested the involvement of claudin-5 protein in a rat OHSS model. These authors showed that the downregulation of claudin-5 expression is a key contributor to the increased ovarian vascular permeability seen in OHSS (Kitajima et al. 2006). In the present study, we also showed that ZO-1 protein levels in endothelial cells incubated with FF from OHSS patients were lower than those of the control group. These results are consistent with those obtained by Levin et al. (1998), who showed that FF from patients suffering from severe OHSS induces destruction of the endothelial ZO-1 TJ protein. We found no changes in ZO-1 levels after the pre-incubation of FF from women at risk of OHSS with the ANGPT1-neutralising antibody. This observation suggests that ANGPT1 is not involved in the regulation of ZO-1 protein in OHSS patients. However, we cannot discard a possible effect of ANGPT1 on other ZO intra-cytoplasmic proteins such as ZO-2 and -3, which are also involved in endothelial TJ.

Besides claudin- 5 and $\mathrm{ZO}$, other proteins are involved in the regulation of endothelial permeability, such as those forming AJ complexes. AJ are mainly composed of transmembrane proteins from the cadherin family and cytosolic catenins. Endothelial cells express a cadherin called vascular endothelial (VE)cadherin, which acts as an important regulator of vascular integrity and permeability (Dejana et al. 2009). In this study, we analysed the expression of VE-cadherin and phospho$\beta$-catenin (active form) in endothelial cells with FF from patients at risk of OHSS. Incubation with FF from patients at risk of OHSS resulted in a significant decrease in endothelial cell content of VE-cadherin. The pre-incubation of the neutralising antibody against ANGPT1 with FF from OHSS patients restored VE-cadherin levels to control values. The FF from OHSS patients caused an increase in the levels of the phosphorylated form of $\beta$-catenin in endothelial cells, in comparison with control patients. The blockage of ANGPT1 in FF from OHSS patients restored the levels of phosphorylated $\beta$-catenin to normal values. Taken together, these results show that the ANGPT1 from patients at high risk of OHSS alters some of the TJ and AJ proteins, which affects endothelial cell interaction and may lead to increased vascular permeability. Our data are consistent with those obtained by other groups in reference to the crosstalk between VE-cadherin and TJ (Gavard and Gutkind 2008; Taddei et al. 2008). The decrease in plasma membrane 
VE-cadherin levels correlates with the disorganisation of the endothelial TJ. In addition, claudin- 5 mRNA is downregulated in endothelial cells that lack VE-cadherin expression. When VE-cadherin is located at AJ between endothelial cells, it blocks $\beta$-catenin and FoxO binding to the claudin- 5 promoter, preventing the transcription of claudin-5. Therefore, VEcadherin directly modulates claudin- 5 expression and, consequently, the organisation and maturation of endothelial TJ (Gavard and Gutkind 2008; Taddei et al. 2008). Regarding this, Hino et al. (2005) showed that $\beta$-catenin is stabilised when phosphorylated at Ser675 by protein kinase A, thus inhibiting its ubiquitination and promoting accumulation of $\beta$-catenin in the nucleus. After nuclear translocation, phospho- $\beta$-catenin regulates transcription of several genes (Bienz and Clevers 2000).

Previous studies have correlated increased levels of vasoactive substances in the vasculature of the ovary with the development of OHSS (Schenker and Polishuk 1976; Leung et al. 1983; Zaidise et al. 1983; Pride et al. 1984; Navot et al. 1987). Previously, we have observed high levels of ANGPT1 in FF from patients at risk of OHSS compared with control patients (Scotti et al. 2013). In the present work, we showed that ANGPT1 in FF from OHSS patients, besides its classical role in periendothelial cells, is partially responsible for the decrease in the expression of endothelial TJ and AJ. This suggests a crucial role of ANGPT1 in the regulation of these endothelial junction proteins in patients at risk of OHSS.

Several authors have observed that the levels of VEGFA in serum and FF are significantly increased in patients who developed OHSS compared with patients who did not (Krasnow et al. 1996; Artini et al. 1998). It should be noted that VEGFA levels in FF are 10 times greater than those detected in the plasma of OHSS patients, suggesting that the dominant site of production of this angiogenic factor is the ovary (Artini et al. 1998). In addition, in a previous study, we demonstrated that ANGPT1 levels not only in FF but also in granulosa-lutein cell cultures from patients at risk of OHSS were higher compared with those from patients who are not at such risk (Scotti et al. 2013). We consider that the ovary is the main source not only of VEGFA but also of ANGPT1 in OHSS and that these factors are able to cross the ovarian basement membrane barrier to reach the blood.

Our results using in vivo and in vitro angiogenesis assays allow a better understanding of some of the biochemical and molecular mechanisms that lead to the increased angiogenesis observed in OHSS. Our findings suggest that ANGPT1 increases pathophysiological angiogenesis in patients who are at risk of OHSS and that one of its mechanisms of action is the regulation of tight and adherens junction proteins.

In conclusion, elucidating the biochemical mechanisms by which ANGPT1 regulates vascular development, cell-cell junctions and their integrity in OHSS will lead to identifying new therapeutic targets for the treatment of many human diseases that display aberrant vascular leakage.

\section{Acknowledgements}

This study was supported by Agencia Nacional de Promoción Científica y Técnica (ANPCyT; PICT 2008-747 and PICT 2010-248) and by the National Research Council of Argentina (CONICET, PIP 1223).

\section{References}

Abramovich, D., Parborell, F., and Tesone, M. (2006). Effect of a vascular endothelial growth factor (VEGF) inhibitory treatment on the folliculogenesis and ovarian apoptosis in gonadotrophin-treated prepubertal rats. Biol. Reprod. 75, 434-441. doi:10.1095/BIOLREPROD.106.051052

Artini, P. G., Monti, M., Fasciani, A., Tartaglia, M. L., D'Ambrogio, G., and Genazzani, A. R. (1998). Correlation between the amount of folliclestimulating hormone administered and plasma and follicular fluid vascular endothelial growth factor concentrations in women undergoing in vitro fertilisation. Gynecol. Endocrinol. 12, 243-247. doi:10.3109/ 09513599809015596

Avraamides, C. J., Garmy-Susini, B., and Varner, J. A. (2008). Integrins in angiogenesis and lymphangiogenesis. Nat. Rev. Cancer 8, 604-617. doi:10.1038/NRC2353

Bai, Y., Cui, M., Meng, Z., Shen, L., He, Q., Zhang, X., Chen, F., and Xiao, J. (2009). Ectopic expression of angiopoietin-1 promotes neuronal differentiation in neural progenitor cells through the Akt pathway. Biochem. Biophys. Res. Commun. 378, 296-301. doi:10.1016/J.BBRC. 2008.11.052

Bérubé, M., Deschambeault, A., Boucher, M., Germain, L., Petitclerc, E., and Guérin, S. L. (2005). MMP-2 expression in uveal melanoma: differential activation status dictated by the cellular environment. Mol. Vis. 11, 1101-1111.

Bienz, M., and Clevers, H. (2000). Linking colorectal cancer to Wnt signalling. Cell 103, 311-320. doi:10.1016/S0092-8674(00)00122-7

Brooks, P. C., Clark, R. A., and Cheresh, D. A. (1994). Requirement of vascular integrin alpha v beta 3 for angiogenesis. Science 264, 569-571. doi:10.1126/SCIENCE.7512751

Brooks, P. C., Silletti, S., von Schalscha, T. L., Friedlander, M., and Cheresh, D. A. (1998). Disruption of angiogenesis by PEX, a noncatalytic metalloproteinase fragment with integrin-binding activity. Cell 92, 391-400. doi:10.1016/S0092-8674(00)80931-9

Carmeliet, P. (2000). Fibroblast growth factor-1 stimulates branching and survival of myocardial arteries: a goal for therapeutic angiogenesis? Circ. Res. 87, 176-178. doi:10.1161/01.RES.87.3.176

Carmeliet, P., Lampugnani, M. G., Moons, L., Breviario, F., Compernolle, V., Bono, F., Balconi, G., Spagnuolo, R., Oosthuyse, B., Dewerchin, M., Zanetti, A., Angellilo, A., Mattot, V., Nuyens, D., Lutgens, E., Clotman, F., de Ruiter, M. C., Gittenberger-de Groot, A., Poelmann, R., Lupu, F., Herbert, J. M., Collen, D., and Dejana, E. (1999). Targeted deficiency or cytosolic truncation of the VE-cadherin gene in mice impairs VEGF-mediated endothelial survival and angiogenesis. Cell 98, 147-157. doi:10.1016/S0092-8674(00)81010-7

Chen, S. U., Chou, C. H., Lin, C. W., Lee, H., Wu, J. C., Lu, H. F., Chen, C. D., and Yang, Y. S. (2010). Signal mechanisms of vascular endothelial growth factor and interleukin-8 in ovarian hyperstimulation syndrome: dopamine targets their common pathways. Hum. Reprod. 25, 757-767. doi:10.1093/HUMREP/DEP432

Davis, S., Aldrich, T. H., Jones, P. F., Acheson, A., Compton, D. L., Jain, V., Ryan, T. E., Bruno, J., Radziejewski, C., Maisonpierre, P. C., and Yancopoulos, G. D. (1996). Isolation of angiopoietin-1, a ligand for the TIE2 receptor, by secretion-trap expression cloning. Cell 87, 1161-1169. doi:10.1016/S0092-8674(00)81812-7

Dejana, E., Tournier-Lasserve, E., and Weinstein, B. M. (2009). The control of vascular integrity by endothelial cell junctions: molecular basis and pathological implications. Dev. Cell 16, 209-221. doi:10.1016/ J.DEVCEL.2009.01.004

Edgell, C. J., McDonald, C. C., and Graham, J. B. (1983). Permanent cell line expressing human factor VIII-related antigen established by hybridisation. Proc. Natl. Acad. Sci. USA 80, 3734-3737. doi:10.1073/PNAS.80. 12.3734

Edgell, C. J., Haizlip, J. E., Bagnell, C. R., Packenham, J. P., Harrison, P., Wilbourn, B., and Madden, V. J. (1990). Endothelium specific 
Weibel-Palade bodies in a continuous human cell line, EA.hy926. In Vitro Cell. Dev. Biol. 26, 1167-1172. doi:10.1007/BF02623694

Eliceiri, B. P., Klemke, R., Stromblad, S., and Cheresh, D. A. (1998) Integrin alpha $\mathrm{v}$ beta 3 requirement for sustained mitogen-activated protein kinase activity during angiogenesis. J. Cell Biol. 140, 1255 1263. doi:10.1083/JCB.140.5.1255

Enskog, A., Henriksson, M., Unander, M., Nilsson, L., and Brannstrom, M. (1999). Prospective study of the clinical and laboratory parameters of patients in whom ovarian hyperstimulation syndrome developed during controlled ovarian hyperstimulation for in vitro fertilisation. Fertil. Steril. 71, 808-814. doi:10.1016/S0015-0282(99)00090-4

Fiedler, U., and Augustin, H. G. (2006). Angiopoietins: a link between angiogenesis and inflammation. Trends Immunol. 27, 552-558. doi:10.1016/J.IT.2006.10.004

Gavard, J., and Gutkind, J. S. (2008). VE-cadherin and claudin-5: it takes two to tango. Nat. Cell Biol. 10, 883-885. doi:10.1038/NCB0808-883

Goede, V., Schmidt, T., Kimmina, S., Kozian, D., and Augustin, H. G. (1998). Analysis of blood vessel maturation processes during cyclic ovarian angiogenesis. Lab. Invest. 78, 1385-1394.

Gómez, R., Soares, S. R., Busso, C., Garcia-Velasco, J. A., Simon, C., and Pellicer, A. (2010). Physiology and pathology of ovarian hyperstimulation syndrome. Semin. Reprod. Med. 28, 448-457. doi:10.1055/S-00301265670

Groten, T., Fraser, H. M., Duncan, W. C., Konrad, R., Kreienberg, R., and Wulff, C. (2006). Cell junctional proteins in the human corpus luteum: changes during the normal cycle and after hCG treatment. Hum. Reprod. 21, 3096-3102. doi:10.1093/HUMREP/DEL286

Hanahan, D., and Folkman, J. (1996). Patterns and emerging mechanisms of the angiogenic switch during tumorigenesis. Cell 86, 353-364. doi:10.1016/S0092-8674(00)80108-7

Hartsock, A., and Nelson, W. J. (2008). Adherens and tight junctions: structure, function and connections to the actin cytoskeleton. Biochim. Biophys. Acta 1778, 660-669. doi:10.1016/J.BBAMEM.2007.07.012

Hazzard, T. M., Molskness, T. A., Chaffin, C. L., and Stouffer, R. L. (1999). Vascular endothelial growth factor (VEGF) and angiopoietin regulation by gonadotrophin and steroids in macaque granulosa cells during the peri-ovulatory interval. Mol. Hum. Reprod. 5, 1115-1121. doi:10.1093/ MOLEHR/5.12.1115

Herr, D., Fraser, H. M., Konrad, R., Holzheu, I., Kreienberg, R., and Wulff, C. (2013). Human chorionic gonadotrophin controls luteal vascular permeability via vascular endothelial growth factor by down-regulation of a cascade of adhesion proteins. Fertil. Steril. 99, 1749-1758. doi:10.1016/ J.FERTNSTERT.2013.01.120

Hino, S., Tanji, C., Nakayama, K. I., and Kikuchi, A. (2005). Phosphorylation of beta-catenin by cyclic AMP-dependent protein kinase stabilises beta-catenin through inhibition of its ubiquitination. Mol. Cell. Biol. 25 , 9063-9072. doi:10.1128/MCB.25.20.9063-9072.2005

Humaidan, P., Quartarolo, J., and Papanikolaou, E. G. (2010). Preventing ovarian hyperstimulation syndrome: guidance for the clinician. Fertil. Steril. 94, 389-400. doi:10.1016/J.FERTNSTERT.2010.03.028

Isachenko, V., Mallmann, P., Petrunkina, A. M., Rahimi, G., Nawroth, F., Hancke, K., Felberbaum, R., Genze, F., Damjanoski, I., and Isachenko, E. (2012). Comparison of in vitro and chorioallantoic membrane (CAM) culture systems for cryopreserved medulla-contained human ovarian tissue. PLoS ONE 7, e32549. doi:10.1371/JOURNAL.PONE.0032549

Itoh, M., Nagafuchi, A., Moroi, S., and Tsukita, S. (1997). Involvement of ZO-1 in cadherin-based cell adhesion through its direct binding to alpha catenin and actin filaments. J. Cell Biol. 138, 181-192. doi:10.1083/ JCB.138.1.181

Jiao, H., Wang, Z., Liu, Y., Wang, P., and Xue, Y. (2011). Specific role of tight junction proteins claudin-5, occludin and ZO-1 of the blood-brain barrier in a focal cerebral ischemic insult. J. Mol. Neurosci. 44, 130-139. doi:10.1007/S12031-011-9496-4
Kanda, T., Numata, Y., and Mizusawa, H. (2004). Chronic inflammatory demyelinating polyneuropathy: decreased claudin-5 and relocated ZO-1. J. Neurol. Neurosurg. Psychiatry 75, 765-769. doi:10.1136/JNNP.2003. 025692

Kitajima, Y., Endo, T., Nagasawa, K., Manase, K., Honnma, H., Baba, T., Hayashi, T., Chiba, H., Sawada, N., and Saito, H. (2006). Hyperstimulation and a gonadotrophin-releasing hormone agonist modulate ovarian vascular permeability by altering expression of the tight junction protein claudin-5. Endocrinology 147(2), 694-699. doi:10.1210/EN.2005-0700

Koos, R. D. (1995). Increased expression of vascular endothelial growth/ permeability factor in the rat ovary following an ovulatory gonadotrophin stimulus: potential roles in follicle rupture. Biol. Reprod. 52 1426-1435. doi:10.1095/BIOLREPROD52.6.1426

Krasnow, J. S., Berga, S. L., Guzick, D. S., Zeleznik, A. J., and Yeo, K. T. (1996). Vascular permeability factor and vascular endothelial growth factor in ovarian hyperstimulation syndrome: a preliminary report Fertil. Steril. 65, 552-555.

Kunzi-Rapp, K., Ruck, A., and Kaufmann, R. (1999). Characterisation of the chick chorioallantoic membrane model as a short-term in vivo system for human skin. Arch. Dermatol. Res. 291, 290-295. doi:10.1007/ S004030050410

Lampugnani, MG (2012). Endothelial cell-to-cell junctions: adhesion and signalling in physiology and pathology. Cold Spring Harb. Perspect. Med. 2, a006528. doi:10.1101/CSHPERSPECT.A006528

Lauffenburger, D. A., and Horwitz, A. F. (1996). Cell migration: a physically integrated molecular process. Cell 84, 359-369. doi:10.1016 S0092-8674(00)81280-5

Leng, T., Miller, J. M., Bilbao, K. V., Palanker, D. V., Huie, P., and Blumenkranz, M. S. (2004). The chick chorioallantoic membrane as a model tissue for surgical retinal research and simulation. Retina 24, 427434. doi:10.1097/00006982-200406000-00014

Leung, P., Ho, Y. B., and Moon, Y. S. (1983). Effect of prolactin in an experimental model of the ovarian hyperstimulation syndrome. Am. J. Obstet. Gynecol. 145, 847-849.

Levin, E. R., Rosen, G. F., Cassidenti, D. L., Yee, B., Meldrum, D., Wisot, A., and Pedram, A. (1998). Role of vascular endothelial cell growth factor in ovarian hyperstimulation syndrome. J. Clin. Invest. 102, 1978-1985. doi:10.1172/JCI4814

Maisonpierre, P. C., Suri, C., Jones, P. F., Bartunkova, S., Wiegand, S. J., Radziejewski, C., Compton, D., McClain, J., Aldrich, T. H., Papadopoulos, N., Daly, T. J., Davis, S., Sato, T. N., and Yancopoulos, G. D. (1997). Angiopoietin-2, a natural antagonist for Tie2 that disrupts in vivo angiogenesis. Science 277, 55-60. doi:10.1126/SCIENCE.277.5322.55

Malik, A. B., Lynch, J. J., and Cooper, J. A. (1989). Endothelial barrier function. J. Invest. Dermatol. 93, 62S-67S. doi:10.1111/1523-1747. EP12581072

Maruo, N., Morita, I., Shirao, M., and Murota, S. (1992). IL-6 increases endothelial permeability in vitro. Endocrinology 131, 710-714.

Menager, C., Vassy, J., Doliger, C., Legrand, Y., and Karniguian, A. (1999). Subcellular localization of RhoA and ezrin at membrane ruffles of human endothelial cells: differential role of collagen and fibronectin. Exp. Cell Res. 249, 221-230. doi:10.1006/EXCR.1999.4481

Nap, A. W., Dunselman, G. A., de Goeij, A. F., Evers, J. L., and Groothuis, P. G. (2004). Inhibiting MMP activity prevents the development of endometriosis in the chicken chorioallantoic membrane model. Hum. Reprod. 19, 2180-2187. doi:10.1093/HUMREP/DEH408

Navot, D., Margalioth, E. J., Laufer, N., Birkenfeld, A., Relou, A., Rosler, A., and Schenker, J. G. (1987). Direct correlation between plasma renin activity and severity of the ovarian hyperstimulation syndrome. Fertil. Steril. 48, 57-61.

Navot, D., Bergh, P. A., and Laufer, N. (1992). Ovarian hyperstimulation syndrome in novel reproductive technologies: prevention and treatment. Fertil. Steril. 58, 249-261. 
Neufeld, G., Cohen, T., Gengrinovitch, S., and Poltorak, Z. (1999). Vascular endothelial growth factor (VEGF) and its receptors. FASEB J. 13, 9-22.

Orvieto, R. (2013). Ovarian hyperstimulation syndrome - an optimal solution for an unresolved enigma. J. Ovarian. Res. 6, 77. doi:10.1186/ 1757-2215-6-77

Otrock, Z. K., Mahfouz, R. A., Makarem, J. A., and Shamseddine, A. I. (2007). Understanding the biology of angiogenesis: review of the most important molecular mechanisms. Blood Cells Mol. Dis. 39, 212-220. doi:10.1016/J.BCMD.2007.04.001

Parborell, F., Abramovich, D., and Tesone, M. (2008). Intrabursal administration of the anti-angiopoietin 1 antibody produces a delay in rat follicular development associated with an increase in ovarian apoptosis mediated by changes in the expression of BCL2-related genes. Biol. Reprod. 78, 506-513. doi:10.1095/BIOLREPROD.107.063610

Parsons-Wingerter, P., Lwai, B., Yang, M. C., Elliott, K. E., Milaninia, A., Redlitz, A., Clark, J. I., and Sage, E. H. (1998). A novel assay of angiogenesis in the quail chorioallantoic membrane: stimulation by bFGF and inhibition by angiostatin according to fractal dimension and grid intersection. Microvasc. Res. 55, 201-214. doi:10.1006/MVRE. 1998.2073

Phillips, H. S., Hains, J., Leung, D. W., and Ferrara, N. (1990). Vascular endothelial growth factor is expressed in rat corpus luteum. Endocrinology 127, 965-967. doi:10.1210/ENDO-127-2-965

Pride, S. M., Ho, Y. B., and Moon, Y. S. (1984). Clinical, endocrinologic and intraovarian prostaglandin $\mathrm{F}$ responses to $\mathrm{H}-1$ receptor blockade in the ovarian hyperstimulation syndrome: studies in the rabbit model. Am. J. Obstet. Gynecol. 148, 670-674. doi:10.1016/0002-9378(84)90771-3

Rodewald, M., Herr, D., Fraser, H. M., Hack, G., Kreienberg, R., and Wulff, C. (2007). Regulation of tight junction proteins occludin and claudin 5 in the primate ovary during the ovulatory cycle and after inhibition of vascular endothelial growth factor. Mol. Hum. Reprod. 13, 781-789. doi:10.1093/MOLEHR/GAM066

Rodewald, M., Herr, D., Duncan, W. C., Fraser, H. M., Hack, G., Konrad, R., Gagsteiger, F., Kreienberg, R., and Wulff, C. (2009). Molecular mechanisms of ovarian hyperstimulation syndrome: paracrine reduction of endothelial claudin 5 by hCG in vitro is associated with increased endothelial permeability. Hum. Reprod. 24, 1191-1199. doi:10.1093/ HUMREP/DEN479

Schenker, J. G., and Polishuk, W. Z. (1976). The role of prostaglandins in ovarian hyperstimulation syndrome. Eur. J. Obstet. Gynecol. Reprod. Biol. 6, 47-52. doi:10.1016/0028-2243(76)90001-0
Scotti, L., Abramovich, D., Pascuali, N., de Zúñiga, I., Oubina, A., Kopcow, L., Lange, S., Owen, G., Tesone, M., and Parborell, F. (2013). Involvement of the ANGPTs/Tie-2 system in ovarian hyperstimulation syndrome (OHSS). Mol. Cell. Endocrinol. 365, 223-230. doi:10.1016/J.MCE.2012.10.022

Suri, C., Jones, P. F., Patan, S., Bartunkova, S., Maisonpierre, P. C., Davis, S., Sato, T. N., and Yancopoulos, G. D. (1996). Requisite role of angiopoietin-1, a ligand for the TIE2 receptor, during embryonic angiogenesis. Cell 87, 1171-1180. doi:10.1016/S0092-8674(00)81813-9

Taddei, A., Giampietro, C., Conti, A., Orsenigo, F., Breviario, F., Pirazzoli, V., Potente, M., Daly, C., Dimmeler, S., and Dejana, E. (2008). Endothelial adherens junctions control tight junctions by VE-cadherinmediated upregulation of claudin-5. Nat. Cell Biol. 10, 923-934. doi:10.1038/NCB1752

Umeda, K., Ikenouchi, J., Katahira-Tayama, S., Furuse, K., Sasaki, H., Nakayama, M., Matsui, T., Tsukita, S., Furuse, M., and Tsukita, S. (2006). ZO-1 and ZO-2 independently determine where claudins are polymerised in tight-junction strand formation. Cell 126, 741-754. doi:10.1016/J.CELL.2006.06.043

Valdes, T. I., Kreutzer, D., and Moussy, F. (2002). The chick chorioallantoic membrane as a novel in vivo model for the testing of biomaterials. $J$. Biomed. Mater. Res. 62, 273-282. doi:10.1002/JBM.10152

Villasante, A., Pacheco, A., Ruiz, A., Pellicer, A., and Garcia-Velasco, J. A. (2007). Vascular endothelial cadherin regulates vascular permeability: implications for ovarian hyperstimulation syndrome. J. Clin. Endocrinol. Metab. 92, 314-321. doi:10.1210/JC.2006-1231

von Otte, S., Paletta, J. R., Becker, S., Konig, S., Fobker, M., Greb, R. R., Kiesel, L., Assmann, G., Diedrich, K., and Nofer, J. R. (2006). Follicular fluid high-density lipoprotein-associated sphingosine 1-phosphate is a novel mediator of ovarian angiogenesis. J. Biol. Chem. 281, 5398-5405. doi:10.1074/JBC.M508759200

Wójciak-Stothard, B., Potempa, S., Eichholtz, T., and Ridley, A. J. (2001). Rho and Rac but not $\mathrm{Cdc} 42$ regulate endothelial cell permeability. J. Cell Sci. 114, 1343-1355.

Zaidise, I., Friedman, M., Lindenbaum, E. S., Askenazi, R., Peretz, B. A., and Paldi, E. (1983). Serotonin and the ovarian hyperstimulation syndrome. Eur. J. Obstet. Gynecol. Reprod. Biol. 15, 55-60. doi:10.1016/0028-2243(83)90297-6

Zehendner, C. M., Librizzi, L., de Curtis, M., Kuhlmann, C. R., and Luhmann, H. J. (2011). Caspase-3 contributes to ZO-1 and Cl-5 tightjunction disruption in rapid anoxic neurovascular unit damage. PLoS ONE 6, e16760. doi:10.1371/JOURNAL.PONE.0016760 